\title{
Laparoscopic splenectomy: how minimal can we make it?
}

\author{
Adetokunbo Fadipe $^{1} \cdot$ David Wilkinson $^{1} \cdot$ Robert Peters $^{1} \cdot$ Catherine Doherty $^{2} \cdot$ Nick Lansdale $^{1,3}$ [D
}

Received: 31 October 2021 / Revised: 8 December 2021 / Accepted: 15 December 2021 / Published online: 13 January 2022

(c) The Author(s) 2022

\begin{abstract}
Aims Laparoscopic splenectomy (LS) is routinely performed in children, however, a large spleen in a small child can pose significant operative challenges. We instigated a highly standardised surgical and anaesthetic approach to LS to minimise surgical trauma and enhance recovery. The aim of this study was to assess the outcomes of this programme.

Methods Prospective study of all LS's performed 2018-2021. Surgical approach was via one $10 \mathrm{~mm}$ and three $5 \mathrm{~mm}$ ports. Early hilar control was accomplished with Hem-o-loks. Splenic retrieval via the $10 \mathrm{~mm}$ incision used finger morcellation within an Espiner EcoSac. Anaesthesia utilised a standardised regime of agents and bupivacaine was infiltrated to the splenic bed and wound sites. Post-operative opiates were minimised. Data are presented as median [IQR].

Results Twenty consecutive children were included. Indications for LS were hereditary spherocytosis $(n=12)$, sickle cell disease $(n=6)$, beta-thalassaemia $(n=1)$ and splenic haemangiomatosis $(n=1)$. Age at surgery was 101 months [75-117] and weight $30 \mathrm{~kg}$ [21-37]. Splenic size was $13.4 \mathrm{~cm}$ [12-14.4]. Operative time was $178 \mathrm{~min}$ [156-185]. There were no open conversions and no significant intra or post-operative bleeding. One patient developed pancreatitis. Median post-operative pain score was 1 [1-3]. Median length of stay was 2 days [2-3].

Conclusion LS is feasible, safe and efficient in smaller children with large spleens. This standardised programme of anaesthesia and surgery based around a core team reliably results in few complications, good analgesia and short length of stay.
\end{abstract}

Keywords Laparoscopic $\cdot$ Splenectomy $\cdot$ Child $\cdot$ Enhanced recovery

\section{Introduction}

Since the early adopters started reporting small case series in the early 1990's laparoscopic splenectomy (LS) has become widely accepted as 'gold standard' practice in children $[1,2]$. However, paediatric LS can pose technical challenges related to the reduced working space found in small children with large spleens, which may in turn increase the risk of bleeding, conversion and a prolonged recovery. Similarly, splenic retrieval may be more complicated in the child with a large spleen and the use of accessory incisions has been reported. Whilst single incision laparoscopic splenectomy (SILS) has

Nick Lansdale

nick.lansdale2@mft.nhs.uk

1 Department of Paediatric and Neonatal Surgery, Royal Manchester Children's Hospital, Manchester, UK

2 Department of Paediatric Anaesthesia, Royal Manchester Children's Hospital, Manchester, UK

3 Faculty of Biology Medicine and Health, The University of Manchester, Manchester, UK been advocated by some, this appears to be associated with longer operative times and A greater risk of intra-operative bleeding [3]. Despite these challenges, LS consistently offers benefits such as low complication rates, improved cosmesis and reduced hospital length of stay when compared to the open operation, albeit with concerns over the risk of intraoperative bleeding and longer operative times $[1,2,4]$.

In this study, we share the experience in our centre of developing a standardized surgical and anaesthetic approach to LS amongst a small team of paediatric upper gastrointestinal surgeons and anaesthetists. The aim of this program was to improve outcomes by minimising the risk of complications, length of stay and post-operative pain.

\section{Materials methods}

All cases of LS performed at Royal Manchester Children's Hospital between March 2018 and August 2021 were identified from prospective records. Data collected included demographics (age, weight and gender) as well as clinical 
data (splenic size on most recent ultrasound and indication for surgery). Outcome measures were operative duration, presence of intra-operative bleeding, presence of post-operative complications, length of hospital stay and maximum recorded post-operative pain scores. Continuous data are presented median and interquartile range (IQR).

\section{Anaesthetic approach}

The standardised anaesthetic approach, developed by a core team of anaesthetic consultants, included intraoperative use of a remifentanil infusion, followed by ketorolac, paracetamol and magnesium sulphate. At the close of anaesthesia, an injection of $2 \mathrm{mg} / \mathrm{kg}$ levobupivacaine is delivered into the splenic bed (and wound sites) via a flexible catheter, alongside oxycodone, with dexamethasone and ondansetron as antiemetics. Post-operatively, the focus turns to early mobilisation and early weaning of opiates. Anticoagulant or anti-platelet agents are not routinely used post-operatively.

\section{Surgical approach}

LS was completed by a core group of three consultant paediatric upper gastrointestinal surgeons. The abdominal approach used four incisions: a curved incision was placed in the skin crease of the umbilicus for the $10 \mathrm{~mm}$ primary port and three $5 \mathrm{~mm}$ ports were placed in the left upper quadrant and epigastrium (Fig. 1).

Initially, the short gastric vessels are divided with an ultrasonic device and the splenic hilum revealed. The main splenic vessels (artery first) are dissected clear and ligated using Hem-o-lok clips to achieve early hilar control [5] (Fig. 2).

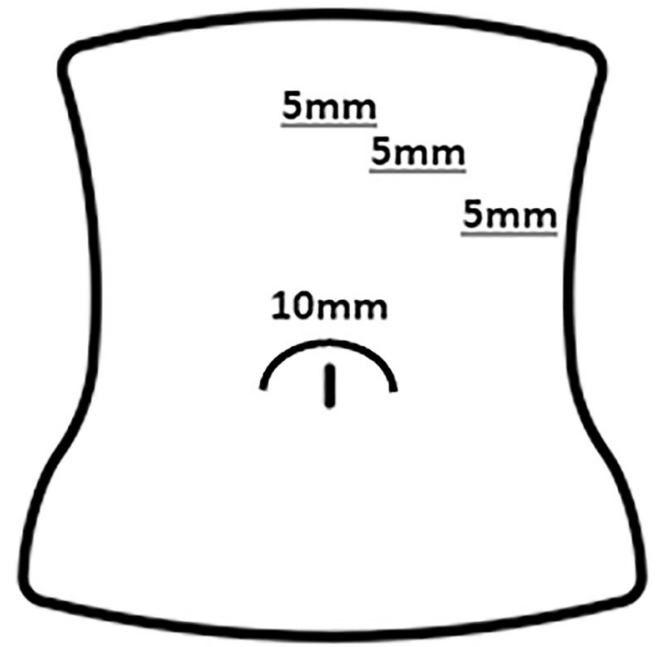

Fig. 1 Surgical incisions used for laparoscopic splenectomy

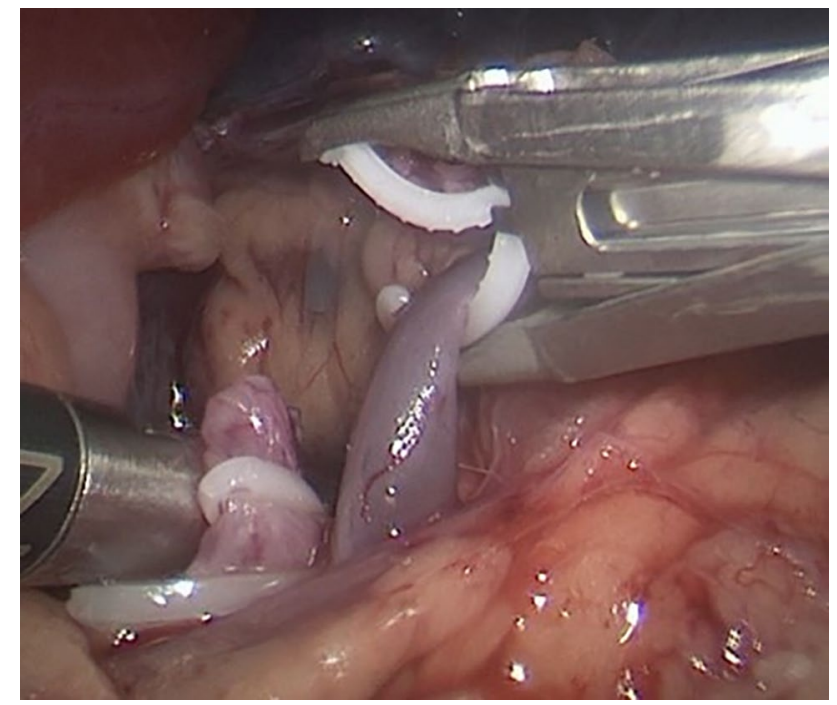

Fig. 2 Ligation of the splenic vein with a Hem-o-lock clip. The splenic artery is seen at the left of the image already ligated

The splenic ligaments are divided with the ultrasonic device and it is confirmed the spleen is completely mobile. To deliver the spleen, the fascia of umbilical incision is extended in the midline superiorly (without extending the skin incision). The spleen is placed in an Espiner EcoSac $170 \mathrm{bag}$ and the edges of this brought out of the umbilical incision. Morcellation of the spleen within the bag is done bluntly using a finger. The spleen is removed piecemeal using Rampley's forceps (Fig. 3).

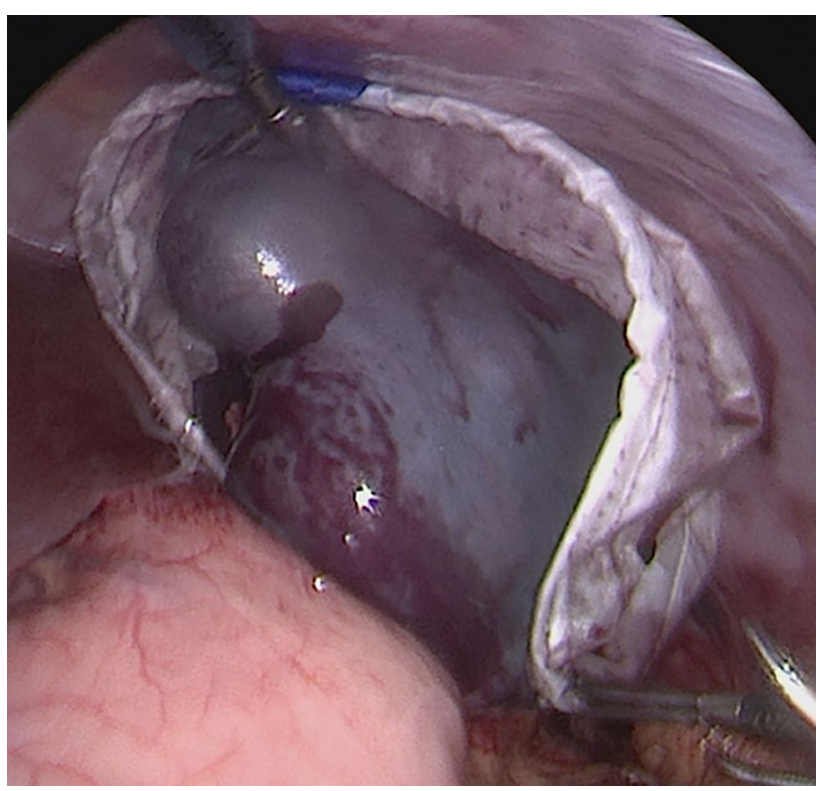

Fig. 3 Placement of the spleen in an Espiner EcoSac 170 bag for morcellation and retrieval 


\section{Results}

There were a total of 20 LS cases (11 female, 9 male) over the three years with a median age of 8.5 years [6.3-9.8]. Weight at the the time of surgery was $30.1 \mathrm{~kg}[21.5-37.4 \mathrm{~kg}]$. Indications for LS, are in Table 1.

Of the 20 cases, six (30\%) included a concomitant laparoscopic cholecystectomy. Median splenic size was $13.4 \mathrm{~cm}$ [12-14.4 cm] as measured in 18 out of 20 cases by preoperative ultrasound. The splenic size to body weight ratio was $0.5 \mathrm{~cm} / \mathrm{kg}[0.4-0.6 \mathrm{~cm} / \mathrm{kg}]$.

\section{Outcomes}

The operative duration for LS only was 178 min [156-185], compared to 184 min with concomitant cholecystectomy. The median length of stay was 2 days [2-3]. There were no conversions to open surgery and no cases of significant intraoperative bleeding. The median maximum pain score was 1 [1-3]. With the anaesthetic regimes utilised, all patients had opiates discontinued within $48 \mathrm{~h}$.

There was one case of prolonged post-operative ileus which resolved spontaneously. Another case had post-operative pain levels disproportionate to their clinical condition. A $\mathrm{CT}$ abdomen was undertaken but the findings were equivocal and visceral injury could not be excluded. A laparotomy was therefore carried out on postoperative day 2: findings were suspicious of pancreatitis but the histology of a resected specimen confirmed only necrotic omentum. There have not been any reports of post-splenectomy sepsis or thrombosis at a minimum follow-up period of $>90$ days.

\section{Discussion}

We have presented a series of LS which includes younger children with comparatively larger spleens and demonstrated good outcomes. The highly standardised technique we have described appears to compare favourably to other large published series, with lower rates of complications and conversion to open surgery [6-9]. Our median

Table 1 Indications for splenectomy

\begin{tabular}{ll}
\hline Indication for laparoscopic splenectomy & $n=(\%)$ \\
\hline Hereditary spherocytosis & $12(60)$ \\
Sickle cell anaemia & $6(30)$ \\
Beta thalassaemia & $1(5)$ \\
Splenic haemangiomatosis & $1(5)$ \\
\hline
\end{tabular}

operative duration of $178 \mathrm{~min}$ is also comparable to these series that demonstrate a range of 120-201 min for LS alone [6-8]. It is therefore important to highlight our lack of any conversions to open surgery did not result in excessively long operative times and demonstrates the technique is efficient.

Major bleeding either during or after LS is the most feared complication for surgeons and whilst others have advocated exclusive use of energy devices for establishing vascular control, we have previously reported our preference for the use of Hem-o-Lok clips and demonstrated them safe for controlling the splenic vessels in LS and with thoracoscopic surgery [5]. In this larger series, we have again found that significant haemorrhage can be avoided in LS. We believe our technique both minimises the risk of intraoperative bleeding and delayed bleeding due to unrecognised thermal injury: we are aware of reports of delayed major haemorrhage with the use of energy devices alone.

There is evidence from Hassan et al. that LS patients tend to resume feeding earlier than their open counterparts, likely contributing to their reduced length of stay [4]. Our series has a short length of stay, with some children even going home the day after surgery. Again, this compares favourably to other larger series [6-9]. We believe the key factor in early discharge is good post-operative analgesia and early mobilisation. Data here show that this technique, incorporating a standardised anaesthetic regime coupled with infiltration of local anaesthetic to the splenic bed and wound sites, achieves excellent post-operative analgesia and permits early discharge. The pain scores reported confirm this, with the median maximum score being 1 and IQR 1-3; we hypothesise these very low levels of reported pain are due to a synergistic effect of reduced surgical trauma, multi-modal, opiate-sparing analgesia and early mobilisation. Although the use of local anaesthetic infiltration to the gall bladder fossa is well described in laparoscopic cholecystectomy, we are unable to find reports of its use in LS in children: whilst unable to prove its efficacy in a controlled manner, we have found it appears very helpful and contributes to the overall efficient analgesia described above.

Low rates of complications, even at the higher splenic sizes have previously been reported: our series reinforces these findings and demonstrates the technique is safe and efficient in the population of smaller children with larger spleens [10]. We believe that in addition to the standardised technique described, good outcomes are achieved by surgeons being experienced in the procedure. In our unit, therefore, a specialised group of three upper gastrointestinal surgeons performs all LS with routine dual consultant operating. This is in-keeping with reports from other centres who have highlighted experienced surgeons as being key to achieving good outcomes [9]. 


\section{Conclusion}

LS is feasible, safe and efficient in smaller children with large spleens. This standardised programme of anaesthesia and surgery based around a core team reliably results in few complications, good analgesia and short length of stay.

Acknowledgments We would like to thank Dr John Grainger, Dr Sabiha Kausar and other members of the Haematology team at Royal Manchester Children's Hospital for guiding the overall care of the patients included in this series.

Author contributions Study conception and design: NL, DW, RP, CD. Data acquisition: AF, CD. Analysis and data interpretation: AF, NL. Drafting of the manuscript: AF. Critical revision: NL, RP, DW, CD.

Funding Not applicable.

Availability of data and material Not applicable.

Code availability Not applicable.

\section{Declarations}

Conflict of interest Not applicable.

Open Access This article is licensed under a Creative Commons Attribution 4.0 International License, which permits use, sharing, adaptation, distribution and reproduction in any medium or format, as long as you give appropriate credit to the original author(s) and the source, provide a link to the Creative Commons licence, and indicate if changes were made. The images or other third party material in this article are included in the article's Creative Commons licence, unless indicated otherwise in a credit line to the material. If material is not included in the article's Creative Commons licence and your intended use is not permitted by statutory regulation or exceeds the permitted use, you will need to obtain permission directly from the copyright holder. To view a copy of this licence, visit http://creativecommons.org/licenses/by/4.0/.

\section{References}

1. Tulman S, Holcomb GW III, Karamanoukian HL, Renhout J (1993) Pediatric laparoscopic splenectomy. J Pediatr Surg 28:689-692

2. Rescorla FJ, Engum SA, West KW et al (2002) Laparoscopic splenectomy has become the gold standard in children. Am Surg 68:297-302

3. Misiakos EP, Bagias G, Liakakos T, Machairas A (2017) Laparoscopic splenectomy: current concepts. World J Gastrointest Endosc 9:428-437. https://doi.org/10.4253/wjge.v9.i9.428

4. Hassan ME, Al Ali K (2014) Massive splenomegaly in children: laparoscopic versus open splenectomy. JSLS. https://doi.org/10. 4293/JSLS.2014.00245

5. Haffenden V, Peters RT, Wilkinson D et al (2021) Hem-o-Lok polymer clips for major vascular control in paediatric minimally invasive surgery. J Ped Endosc Surg 3:101-105. https://doi.org/ 10.1007/s42804-021-00094-w

6. Wood JH, Partrick DA, Hays T et al (2011) Contemporary pediatric splenectomy: continuing controversies. Pediatr Surg Int 27:1165-1171. https://doi.org/10.1007/s00383-011-2929-

7. Qureshi FG, Ergun O, Sandulache VC et al (2005) Laparoscopic splenectomy in children. JSLS 9:389-392

8. Murawski M, Patkowski D, Korlacki W et al (2008) Laparoscopic splenectomy in children-a multicenter experience. J Pediatr Surg 43:951-954. https://doi.org/10.1016/j.jpedsurg.2007.11.040

9. Patkowski D, Chrzan R, Wróbel G et al (2007) Laparoscopic splenectomy in children: experience in a single institution. J Laparoendosc Adv Surg Tech 17:230-234. https://doi.org/10.1089/lap. 2006.0058

10. Karadag CA, Erginel B, Kuzdan O et al (2015) Impact of spleen size on outcomes in laparoscopic splenectomy in children. Gastroenterol Res Pract. https://doi.org/10.1155/2015/603915 